\title{
Refractivity Measurements on Canada Balsam by Interferometry
}

\author{
James B. Saunders
}

\begin{abstract}
The index of refraction of three different samples of Canada balsam, each obtained from a different source, was measured for thirteen spectral lines in the visible range by means of the Fabry-Perot interferometer. This instrument permits absolute measurements when the method of coincidence (or method of exact fractions) is applied to fringes produced in thin films of the material being tested. High accuracy may be obtained by applying the method to fringes produced in films of successively increasing thickness.
\end{abstract}

\section{Introduction}

The increasing requirements for precision optical tools have expanded the need for more precise measurements on the refractive index of optical cements. The author was faced with this problem in the design of a "Kösters interferometer", 1 in which a film of optical cement appears in one of the component light beams. If white light is to be used, as was desired, the film of cement should be compensated for. This is easily effected if the two prisms, required for this instrument are made of glass that has optical properties similar to the cement used to bind them together. In order to select a suitable glass-cement combination it is necessary that the optical properties be sufficiently well known.

The basic principle used for these measurements is the determination of optical and geometrical thicknesses of a nearly parallel film by interference of light. This method of measuring refractive indices was selected because of its convenience and applicability to the measurements of indices for the many spectral lines desired. It is suitable for all transparent liquids as well as other materials that may be molded into optical films or wedges. Fair accuracy is obtainable on thin films, such as those usually used for cementing optical elements, and increasing accuracy is obtainable with films of increasing thickness. Several spectral lines are used for applying the "method of coincidence", and values are obtained for each of the lines used. An accuracy of five in the fourth decimal place of index is obtainable with a single-specimen assembly, forming a film $0.1 \mathrm{~mm}$ thick, if the sample is completely unknown. An increase over the abovementioned accuracy, by a factor of one hundred, is obtainable with a second specimen, if a most favorable choice of film thickness is made and temperatures are sufficiently well-controlled. The preparation of a sample that is completely unknown differs somewhat from that of a sample known to an accuracy of one or better in the second decimal place of index. If an accuracy exceeding five in the fourth decimal place is desired on an unknown

\footnotetext{
1 Werkstatts Technik und Werksleiter 23, 23 (1938).

2 A. Perot and Chas. Fabry, Méthodes interférentielles pour la mesure des grandes epaisseurs et la comparison des longuers d'onde, Ann. chim. et phys. 16, $289(1899)$.
}

sample, two or more specimens are mounted concurrently to insure homogeneity of index from one specimen to the other. The ultimate accuracy obtainable by this method is limited chiefly by temperature control, variations in index among the several assembled specimens, and the accuracy of the wavelength of the light used. Temperature appears to be the dominant factor.

\section{Method of Test}

\subsection{Temperature Considerations}

A change of $1 \mathrm{deg} \mathrm{C}$ in the temperature of Canada balsam produces a change of from 2 to 5 (depending upon wavelength) in the fourth decimal place of refractive index. The temperatures of the specimen varied between $26^{\circ}$ and $28^{\circ} \mathrm{C}$, but were measured to better than $0.1 \operatorname{deg} \mathrm{C}$ and the results corrected to $27^{\circ} \mathrm{C}$. Temperature coefficients, sufficiently accurate for applying the method of coincidence, were obtained for a few spectral lines by observing the changes in orders of interference for measured temperature changes. The coefficients used in computing the final results, however, were values resulting from a statistical analysis of all the data.

\subsection{Mounting of Specimens}

Specimens of three samples of Canada balsam were cooked to a consistency considered proper for use by an experienced optical worker. As an accuracy of one in the fourth decimal place was considered adequate, two specimens of each of the three samples were mounted as follows: Two rectangular plates of glass (see fig. 1,A), approximately 2 by 7 in., with good optical surfaces, were each coated on one side with a partially transmitting, high-reflecting coat of aluminum. Samples from each of the three cements were drawn to long thin threads and placed parallel to each other on one of the coated surfaces and parallel to the long sides. Two spacers, shown in figure $1, \mathrm{~A}$, that are approximately $0.1 \mathrm{~mm}$ thick and equal to within $0.001 \mathrm{~mm}$, were also placed on this surface, and the second coated surface was placed on the three specimens. This combination 

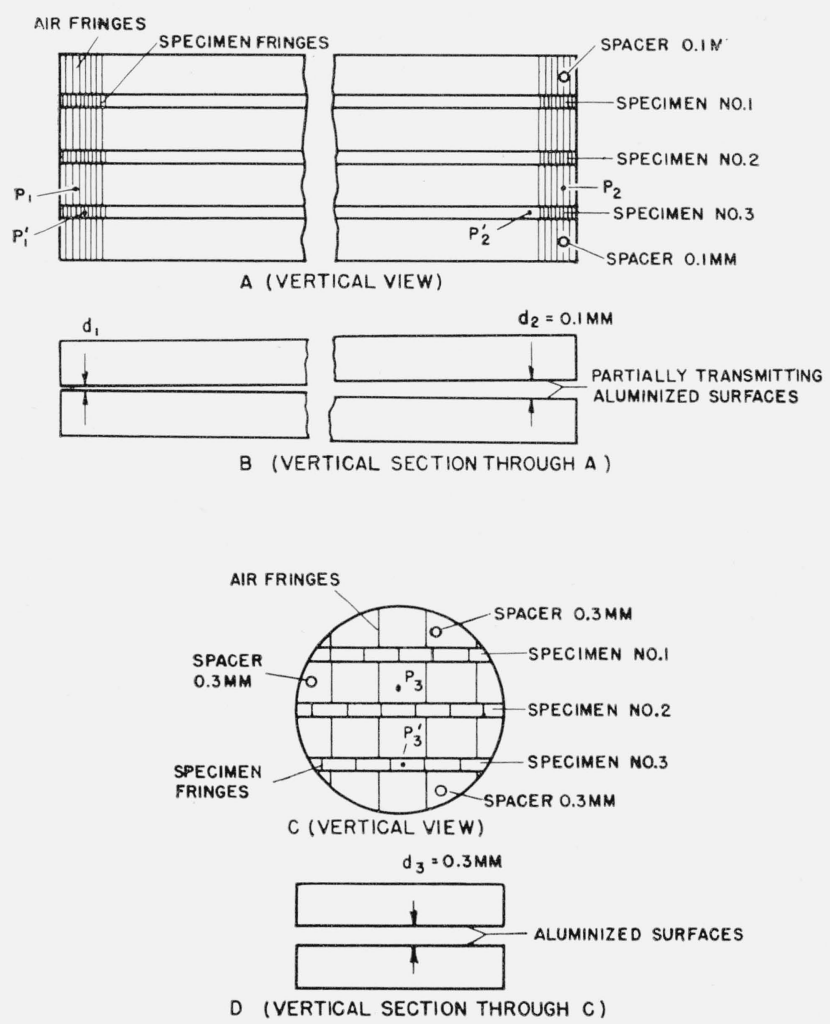

FIGURE 1. Schematic representation of interferometer plates.

F $A$ represents the assembly of the two 2- by 7 -in. Fabry-Perot interferometer plates forming a wedge, with the two spacers, $S_{1}$ and $S_{2}$, controlling the angle. The three specimens conform to the wedge angle. The thickness of the wedge approximates zero at one end of the plates and the thickness of the two equal approximates zero at one end of the plates and the thickness of the two equal spacers at the other; $B$ is a vertical section through $A ; C$ is similar to assembly
$A$, except that three spacers are used to form a thicker and nearly parallel film with a specific direction for the refracting edge of the wedge; $D$ is a vertical section through $C$

was then placed in an oven and heated to a temperature at which the cement softens and fuses to the aluminized surfaces. A rigid body weighing several ounces was heated with the plates. This weight was then placed on the top plate to press the cement threads into narrow ribbons that became quite thin at one end and approximately to the thickness of the two spacers at the other end.

A second set of specimens, one from each of the three samples of cement, was drawn to threads larger than those of the set shown in figure $1, \mathrm{~A}$, and placed parallel to each other on circular fla í plates, each of which was also coated with a partially transmitting film of aluminum. The circular plates were $1 \mathrm{in}$. in diameter. Three spacers are placed on this film, as shown in figure 1,C. The relative thicknesses of these spacers were previously adjusted by grinding to produce a most favorable wedge (approximately three fringes of green light) for reading the interference fringes when they serve as spacers between the two Fabry-Perot interferometer plates. The thickness of these spacers was approximately $0.3 \mathrm{~mm}$. In order to apply the method of exact fractions the approximate thickness at the reference point is meas- ured to within $0.01 \mathrm{~mm}$. This can be obtained with a good micrometer. A second plate, similar to the one on which these last specimens were mounted, was then placed on the specimens and the assembly placed in the furnace, along with the previously described assembly. These specimens are also pressed into ribbons by means of a weight on the top plate. The thickness of these ribbons of Canada balsam will approximate that of the three spacers. If the relative thicknesses of these spacers are properly adjusted, the fringes of interference, formed by light reflected from the highly reflecting surfaces when the plates are assembled into a Fabry-Perot interferometer, will be nearly normal to the ribbons. This orientation is most favorable for reading the orders of interference. A small wedge between the reflecting surfaces, producing approximately five fringes, is also quite favorable to reading the orders of interference. With these most favorably orientated and spaced Fabry-Perot fringes, the fractional orders of interference of the several spectral lines can be measured to one-hundredth. For a thickness of $0.3 \mathrm{~mm}$ this corresponds to approximately one in the fifth decimal place of index. An error of $0.01 \mathrm{~mm}$ in the observed order of interference is, therefore, negligible. When the two assemblies are cooled to ambient temperatures the cement binds the plates together for convenience of handling.

\subsection{Reading of Fringes}

The rectangular-plate assembly was mounted vertically, with the specimen ribbons running horizontally, on a small adjustable bench milling-machine base. A collimated beam of light, from a monochromator and line source, was projected normally through the surfaces. The light was received with a low-power, short-focus telescope that was focused on the ribbon films. Multiple-beam-reflection interference fringes were observed in each of the specimens and also in the spaces between them. For purpose of discussion the fringes seen between and outside the area of the specimens will be called "air fringes," and those inside the area of the specimens, "specimen fringes."

The milling-machine base permits measurable movements of the plate in the focal plane of the telescope. Thus, a panoramic view of the whole plate is observable in the telescope, and the micrometer permits accurate measurements to be made on the fractional orders of interference.

The orders of interference of air fringes for all wavelengths of light used were determined at $P_{1}$, where the separation of the plates is $d_{1}$, by means of the method of coincidence. This is an easy and positive test because $d_{1}$ is known to be very small. With all orders of interference known at $P_{1}$, the orders of interference are determined at $P_{2}$ by counting the number of fringes between these two points and adding them to the order values at $P_{1}$. The narrowness of the samples, with the fringes nearly normal to their lengths, permits a determination of the orders of the air fringes interpolated to points 
$P_{1}^{\prime}$ and $P_{2}^{\prime}$, although these points are in the region of the specimen fringes. If the reflecting surfaces are plane (resulting in straight fringes), the order of interference, at a point in the wedge that is adjacent to but outside the area of the fringes, may be accurately measured with a moveable reticule in the eye piece of the viewing instrument. ${ }^{3}$

The number of specimen fringes between points $P_{1}^{\prime}$ and $P_{2}^{\prime}$ is obtained by counting as the plates are moved, with the adjustable support, so as to cause $P_{2}^{\prime}$ to move into the position of $P_{1}{ }^{\prime}$. The ratio of the number of specimen fringes to the number of air fringes between $P_{1}^{\prime}$ and $P_{2}^{\prime}$ is the refractive index of the specimen relative to air. This ratio is also equal to the corresponding ratios of orders of interference at any point. As the order is low at $P_{1}$ (10 to 20 in the case of this test), the computed order for the specimen fringes at $P_{1}^{\prime}$ will give the integral part of the order number to absolute accuracy, and the fractional part is observed directly. The order at $P_{1}^{\prime}$ plus the number of fringes between $P_{1}^{\prime}$ and $P_{2}{ }^{\prime}$ is the absolute order at $P_{2}^{\prime}$. The ratio of orders of specimen and air fringes at $P_{2}{ }^{\prime}$ is the accepted value for the refractive index of the specimen obtainable with this assembly.

By using the above procedure, the refractive index is obtained for all spectral lines used. A similar treatment also permits measurements for the other two specimens. The order number of the air fringes at $P_{2}^{\prime}$ was approximately $400 \quad(\lambda=0.5 \mu)$, and the fraction is known to one-tenth, even though these narrow fringes are unfavorable for best precision. This, however, corresponds to a precision of about four in the fourth decimal place of refractive index.

The accuracy of index values, determined by this method and assuming constant temperature control, depends upon the thickness of the specimen and the accuracy with which the fractional parts of the orders can be determined. The second set of specimens, assembled concurrently with the set discussed above and shown in figure 1, C, permit an increased accuracy of the measured indices. The rectangular plates are replaced by the circular plates on the adjustable base, and the order numbers for the air fringes are determined at some chosen point, $P_{3}$, by means of the method of exact fractions. The thickness of the air film at $P_{3}$ is approximately three times that at $P_{2}$, consequently the computed orders for the specimen fringes at $P_{3}{ }^{\prime}$, using the results obtained at $P_{2}{ }^{\prime}$, will be in error by not more than three-tenths of a unit. The integral part is, therefore, determined with absolute certainty. As the fringes are very narrow relative to their spacing and as a most favorable spacing was chosen, the errors in the fractional parts are believed to be less than one-hundredth of a unit. The order number at $P_{3}^{\prime}$ was approximately $1,200 \quad(\lambda=0.5 \mu)$. Consequently, errors in the refractive indices, resulting from the order determinations, are negligible compared to those resulting from temperature.

\section{Results}

Three different samples of Canada balsam, each obtained from different places, were measured for 13 visible spectral lines, distributed as uniformly as available sources permitted. Sample 1 had been bleached for a prolonged period by continuous outdoor exposure over a period of approximately 2 years in direct sunlight and was almost free from color. Sample 2 was light yellow and is believed to have been chemically bleached. Sample 3 was dark brown and unbleached. All three were in liquid form.

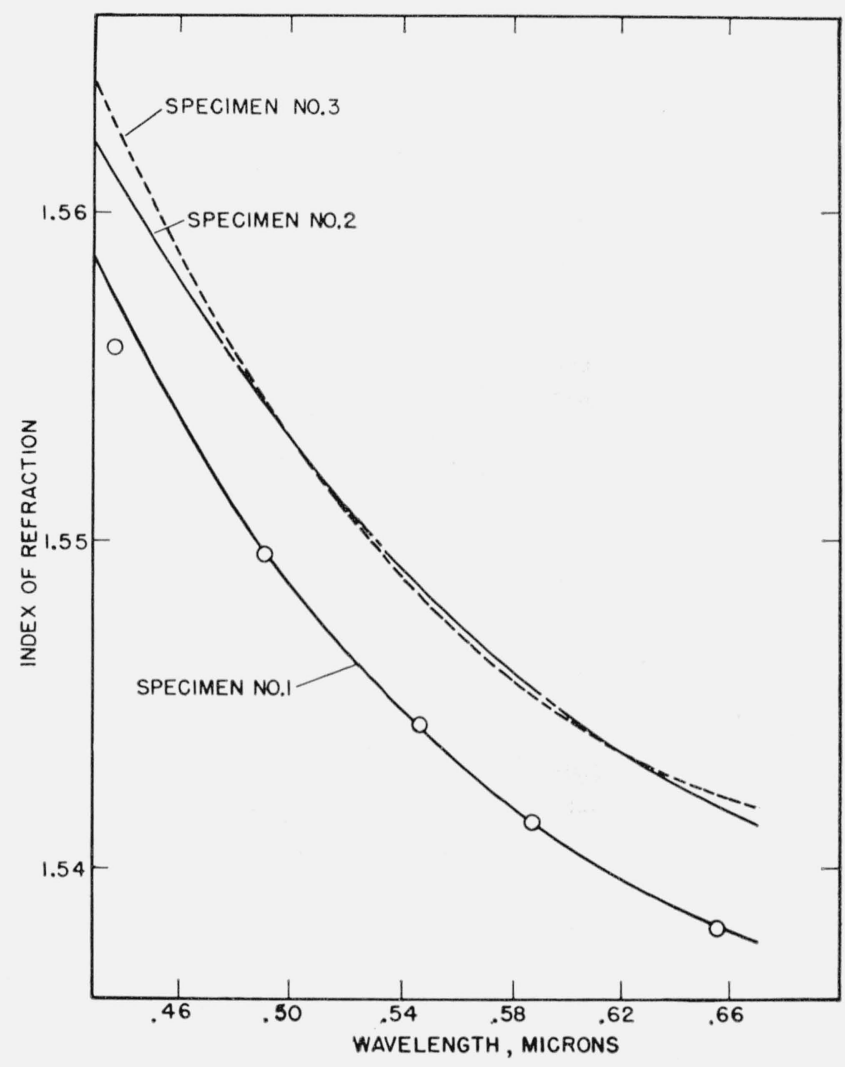

Figure 2. Graphical representation of results.

The curves represent the refractive index of three samples of Canada balsam. The circles represent discrete values for an available optical glass, chosen as most similar to that of specimen 1 .

The three curves in figure 2 represent the most probable index values, corrected to $27^{\circ} \mathrm{C}$. The values for these curves were computed from an equation relating index to wavelength and temperature. The parameters were evaluated statistically. The computed dispersions ( $\mu$-values) for samples 1,2 , and 3 are 43.5, 41.1, and 42.4, respectively. The circles in figure 2 represent index values for an available optical glass. Except for short wavelengths, specimen 1 is seen to be quite adequate for the construction of a Kösters interferometer prism from the glass represented.

Washington, July 15, 1954. 